\title{
Population genomic screening of all young adults in a health-care system: a cost-effectiveness analysis
}

\author{
Lei Zhang, $\mathrm{PhD}^{1,2}$, Yining Bao, $\mathrm{BSc}^{2,3}$, Moeen Riaz, $\mathrm{PhD}^{2}$, Jane Tiller, MgenCouns ${ }^{2}$, Danny Liew, $\mathrm{PhD}^{2}$, \\ Xun Zhuang, $\mathrm{PhD}^{3}$, David J. Amor, $\mathrm{PhD}^{4}$, Aamira Huq, MD ${ }^{5}$, Lara Petelin, $\mathrm{LLB}^{6}$, Mark Nelson, MD", \\ Paul A. James, $\mathrm{MD}^{5}$, Ingrid Winship, $\mathrm{MD}^{5}$, John J. McNeil, $\mathrm{PhD}^{2}$ and Paul Lacaze, $\mathrm{PhD}^{2}$
}

\begin{abstract}
Purpose: To consider the impact and cost-effectiveness of offering preventive population genomic screening to all young adults in a single-payer health-care system.

Methods: We modeled screening of 2,688,192 individuals, all adults aged $18-25$ years in Australia, for pathogenic variants in BRCA1/BRCA2/MLH1/MSH2 genes, and carrier screening for cystic fibrosis (CF), spinal muscular atrophy (SMA), and fragile $\mathrm{X}$ syndrome (FXS), at $71 \%$ testing uptake using per-test costs ranging from AUD $\$ 200$ to $\$ 1200$ ( USD $\$ 140$ to $\$ 850$ ). Investment costs included genetic counseling, surveillance, and interventions (reimbursed only) for at-risk individuals/couples. Cost-effectiveness was defined below AUD\$50,000/DALY (disability-adjusted life year) prevented, using an incremental cost-effectiveness ratio (ICER), compared with current targeted testing. Outcomes were cancer incidence/mortality, disease cases, and treatment costs reduced.
\end{abstract}

Results: Population screening would reduce variant-attributable cancers by $28.8 \%$, cancer deaths by $31.2 \%$, and CF/SMA/FXS cases by $24.8 \%$, compared with targeted testing. Assuming AUD $\$ 400$ per test, investment required would be between 4 and 5 times higher than current expenditure. However, screening would lead to substantial savings in medical costs and DALYs prevented, at a highly cost-effective ICER of AUD\$4038/DALY. At AUD\$200 per test, screening would approach cost-saving for the health system (ICER = AUD\$22/DALY).

Conclusion: Preventive genomic screening in early adulthood would be highly cost-effective in a single-payer health-care system, but ethical issues must be considered.

Genetics in Medicine (2019) 21:1958-1968; https://doi.org/10.1038/s41436019-0457-6

Keywords: population genomic screening; prevention; costeffectiveness analysis; cancer; preconception carrier screening

\section{INTRODUCTION}

Genomic screening of the adult population has significant potential to prevent disease. Some single-payer or statefunded health-care systems are already considering offering preventive population genomic screening to all their members. ${ }^{1,2}$ A combined genomic screening approach, testing for multiple conditions concurrently, is likely to be most cost-effective, rather than screening for individual, often rare, genetic conditions in isolation. Combined genomic screening is an intuitive concept, yet the necessary cost-effectiveness analysis (CEA) of population screening for multiple conditions concurrently has been lacking. Most modeling of adult genetic screening, to date, has been limited to single genetic conditions, often considered in targeted, high-risk populations, ${ }^{3-6}$ rather than the general population.

Two areas where adult population genomic screening may have the most preventive potential are cancer gene testing, to identify individuals with high risk of common and penetrant forms of familial cancer, and preconception carrier screening (PCS), to address the burden of severe, childhood-onset genetic conditions. In both of these areas, clinical guidelines, standard of care, and health system-funded interventions are available in Australia for at-risk individuals/couples. To our knowledge, the two screening models have never been combined in CEA, nor implemented in population screening together. Preventive genomic screening would be most beneficial if offered in early adulthood, above the age for informed consent, but prior to the

\footnotetext{
${ }^{1}$ Department of Epidemiology and Biostatistics, School of Public Health, Xi'an Jiaotong University Health Science Center, Xi'an, Shaanxi, PR China; ${ }^{2}$ Department of Epidemiology and Preventive Medicine, School of Public Health and Preventive Medicine, Monash University, Melbourne, VIC, Australia; ${ }^{3}$ School of Public Health, Nantong University, Nantong, Jiangsu, China; ${ }^{4}$ Victorian Clinical Genetics Services; Murdoch Children's Research Institute; Department of Paediatrics, University of Melbourne, Royal Children's Hospital, Parkville, VIC, Australia; ${ }^{5}$ Department of Genomic Medicine, Royal Melbourne Hospital; Department of Medicine, Royal Melbourne Hospital, University of Melbourne, Melbourne, VIC, Australia; ${ }^{6}$ Familial Cancer Centre, Peter MacCallum Cancer Centre, Melbourne, VIC, Australia; ${ }^{7}$ Discipline of General Practice, University of Tasmania, Hobart, TAS, Australia. Correspondence: Paul Lacaze (paul.lacaze@monash.edu)
} 


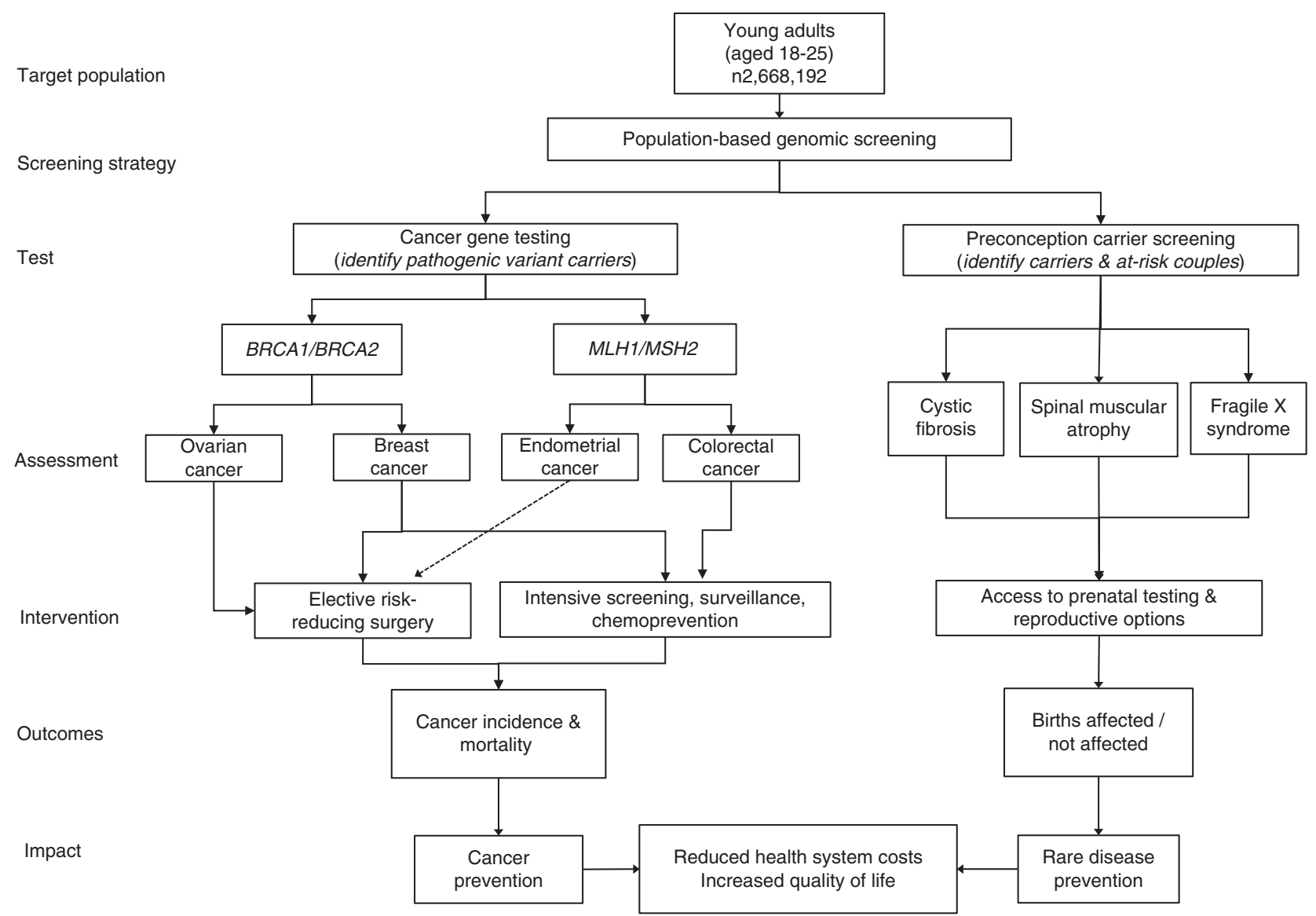

Fig. 1 Schematic: Preventive genomic screening of all young adults in a single-payer health-care system. The model is purposely conservative, based on the Australian health system, estimating the total preventive health impact and cost-effectiveness of offering combined cancer gene testing and preconception carrier screening concurrently to all adults aged 18-25 years.

average age of onset for most hereditary cancer syndromes, and prior to the average age of first childbirth.

The current model of family history or clinical criteria-based gene testing for hereditary cancer is failing to reach many at-risk individuals who need it most, especially younger individuals. Targeted testing for BRCA1/2 has been reported to miss over $50 \%$ of at-risk individuals, even in highrisk communities with increased prevalence of founder variants. ${ }^{4,7}$ One study estimated family history-based testing, at the current rate, would take 250 years to identify all BRCA1/2 carriers in the UK population. ${ }^{9}$ Detection rates for Lynch syndrome (predisposition to colorectal, endometrial, and other cancers) are even lower, with an estimated $95 \%$ of individuals in Australia remaining undetected. ${ }^{10}$ This is despite the proven, and sometimes life-saving, interventions available. ${ }^{11}$ Although the prevalence of hereditary cancers is often considered rare, identification of affected families at the population level has a large preventive impact.

CEA of population-based cancer gene testing has mostly been in the context of hereditary breast and ovarian cancer (HBOC), ${ }^{4,5,12}$ where cost-effectiveness has been demonstrated in the US/UK unselected female population aged $>30$ years. ${ }^{13}$ More recently, some of the first real-world population-based HBOC screening programs have been implemented. This includes screening of 50,726 unselected adults in a single USbased health-care system, identifying five times as many atrisk individuals than previous clinical care. ${ }^{14}$ Over $80 \%$ of identified at-risk individuals had received no prior clinical testing, despite having actionable $B R C A 1 / 2$ pathogenic variants. A second study screened 5908 unselected Australian women with a positive detection rate of $0.64 \%$ (ref. ${ }^{15}$ ). This study found $42 \%$ of identified at-risk women did not have a firstdegree relative with breast or ovarian cancer, and would not meet the current eligibility for funded testing.

CEA of population-based testing for Lynch syndrome, ${ }^{16}$ and the return of clinically actionable variants for adult-onset conditions,${ }^{17}$ has also been conducted. CEA of preconception carrier screening has been mainly limited to single diseases, predominantly cystic fibrosis. ${ }^{6}$ CEA for combined carrier screening of multiple conditions has been limited, ${ }^{18}$ despite the availability of expanded carrier testing panels and recent calls for such work. ${ }^{19}$

The Human Genetics Society of Australasia, American College of Medical Genetics and Genomics, and UK Human Genetics Commission support offering carrier screening for recessive conditions with high carrier frequencies and severity. ${ }^{20-22}$ Yet estimated rates of preconception carrier screening for conditions such as cystic fibrosis, spinal muscular atrophy, 
and fragile X syndrome remain below 5\% in Australia, with testing not reimbursed by the public health-care system. ${ }^{23}$

Here we present, for the first time, a combined CEA of preventive genomic screening for seven different genetic conditions concurrently, offered universally in early adulthood through a single-payer health-care system (Fig. 1 schematic). We model screening from the health system perspective, to reflect the real and emerging possibility of large health systems funding genomic testing at the population level, for prevention and cost reduction. We focus only on genetic conditions with an existing evidence base to justify consideration for population screening, where early identification of risk has high clinical impact and reimbursed health services are currently available. Sufficient health system data are available for these conditions to forecast the costs and likelihood of uptake of interventions, following genetic testing. We purposely modeled cost-effectiveness in this limited set of conditions, as a proof-of-concept toward more expansive genomic screening in the future.

\section{MATERIALS AND METHODS}

This study evaluated the impact on lifetime costs and clinical consequences of offering preventive population genomic screening to all adults aged 18-25 years in Australia, assuming a $71 \%$ testing uptake, ${ }^{13}$ compared with current estimated rates of targeted testing (15\% for cancer gene testing ${ }^{12}$ and $5 \%$ for preconception carrier screening ${ }^{23}$ ). For model design, inputs, and calculations, see Supplementary Materials.

\section{Decision tree models}

Seven independent decision-analytic models were constructed to comprise the genomic screen (Fig. 2): cancer gene testing for breast, ovarian, colorectal, and endometrial cancer; and preconception carrier screening for CF, SMA, and FXS. Decision trees illustrate the alternative choices, interventions and outcomes for individual/couples after testing, based on Australian health system data and published literature (see Supplementary Materials S2.1-2.7).

For cancer gene testing, the prevalence ${ }^{24,25}$ and penetrance $^{26,27}$ of pathogenic variants were used to estimate the incidence of cancer in the population attributable BRCA1/2 and $M L H 1 / M S H 2$ genes. Weighted average lifetime risk (penetrance) was used for BRCA1/2 combined, and $M L H 1 /$ $\mathrm{MSH} 2$ combined, rather than for individual genes. We assumed $100 \%$ testing sensitivity (assumptions Table S4).

For preconception carrier screening (PCS), frequency of carriers, at-risk couples, and affected pregnancies for $\mathrm{CF}+$ SMA + FXS were modeled using European-based carrier frequencies and the national lifetime fertility rate per woman. For FXS, only females with repeat size $>70$ were considered carriers, with weighted risk of maternal transmission calculated per repeat size. A total of 2,339,339 births were estimated in the population lifetime, considered as a "closed population", with no migration.

\section{Model inputs}

We collected model indicators across four categories: demographic, disease-related, costing, and life quality information (Table S1-S3). Demographic information included population size, fertility rate, and life expectancy, sourced from the Australian Bureau of Statistics. Disease-related data included population frequency of conditions, penetrance of cancer genes, disease survival rates, impact of current targeted testing, and surveillance and treatment in the Australian health-care system.

Costs of genomic screening, confirmation testing, and subsequent surveillance and treatment were obtained from the literature or government reports (Table S2). The costs and impact of prevention achieved through chemoprevention and risk-reducing surgery were calculated. Post-diagnosis cancer treatment was averaged to an estimate of AUD\$33,400 per year, across all invasive cancer types (Australian Institute of Health and Welfare, 2017). Burden of disease was measured in disability-adjusted life years (DALYs), with one DALY equivalent to the loss of one year of healthy life due to diseaseinduced disability. Disability weights for cancer were obtained from the Global Burden of Disease Study, ${ }^{28}$ but were not available for CF/SMA/FXS, so Down syndrome was used a proxy (0.81), as done previously. ${ }^{29}$

\section{Impact evaluation and cost-effectiveness analyses}

Decision-analytic models evaluated population genomic screening for seven conditions (S2.1-2.7). Each model was assessed independently, and then in combinations. We modeled four scenarios: i) each condition independently; ii) combined groups- breast and ovarian cancer, colorectal and endometrial cancer (Lynch syndrome), and combined PCS; iii) all cancers combined and combined PCS; and iv) all seven conditions combined into a single test.

We repeated these four scenarios for different per-test screening prices (AUD $\$ 200 / \$ 400 / \$ 800 / \$ 1200$ ), guided by benchmarks including the current government-reimbursed price for HBOC testing in Australia (AUD\$1200) and the prepair $^{\text {TM }}$ carrier screen (AUD\$385) (ref. ${ }^{23}$ ). There were a total of 16 scenarios. We present findings at AUD\$400 per test, a price considered feasible, and AUD\$200, feasible in the near future.

Impact of population cancer gene testing was measured in reduced cancer incidence/mortality, achieved by early surveillance and chemoprevention for pathogenic variant carriers, and elective risk-reducing surgeries for some (conservative uptake estimates, Table S3). Impact of preconception carrier screening was measured by reduction in disease cases, through prenatal diagnosis (PND) (0.8 uptake) and elective termination of affected pregnancies (TOP) (0.5 uptake). We modeled subsequent probability of unaffected future pregnancy, no pregnancy, or affected pregnancy $(0.65$, 0.30 , and 0.05 respectively), to calculate the number of unaffected births following screening (and DALYs prevented).

Total investment was calculated by adding the costs of screening, confirmation testing, genetic counseling, ongoing 


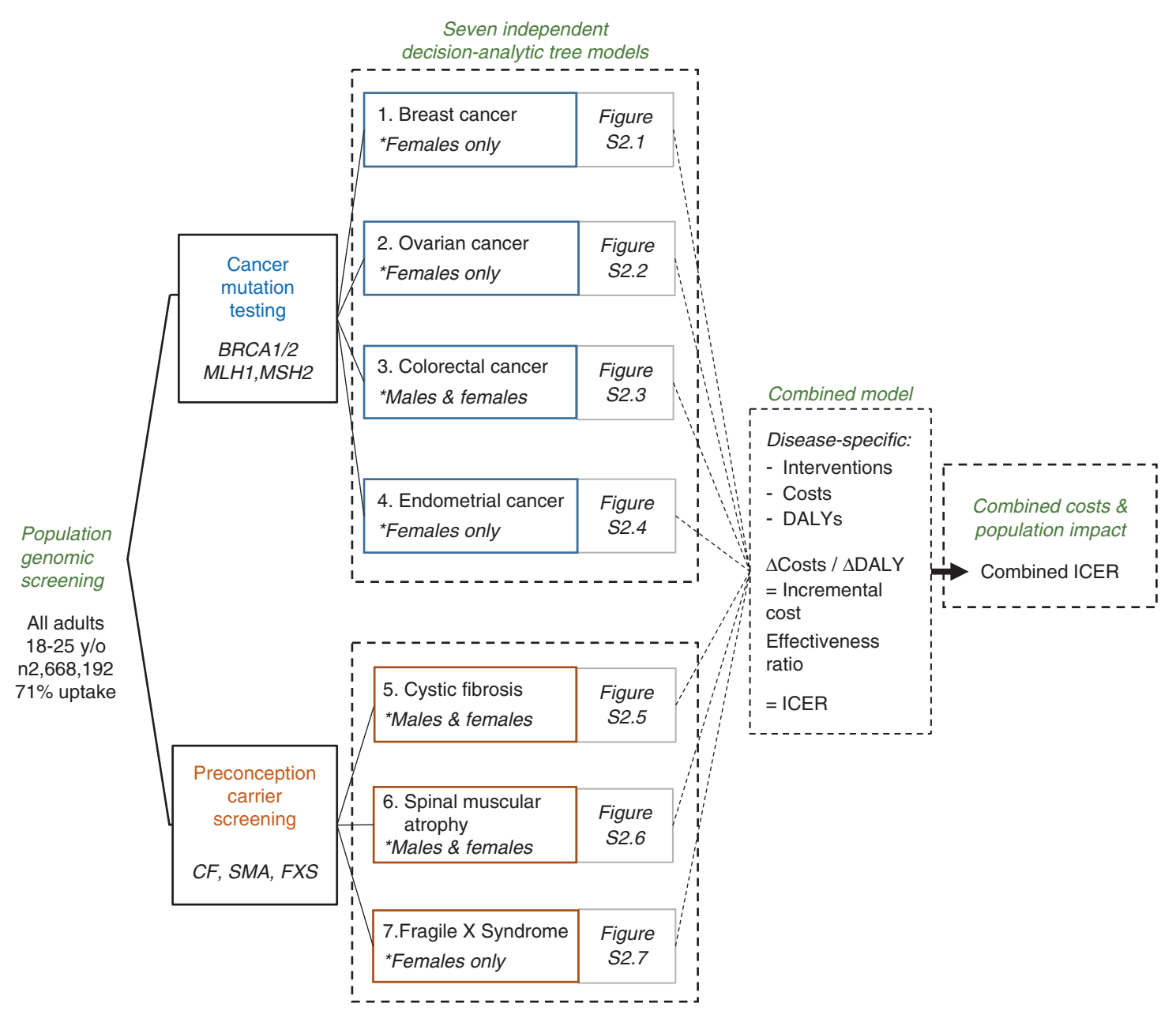

Fig. 2 Population genomic screening: decision-analytic tree models. Seven independent decision-analytic tree models were constructed. Each forecasts the cost-effectiveness and preventive impact of population genomic screening for a given disease, compared with current targeted/criteria-based testing. Models focused on either adult cancer gene testing (S2.1-2.4) or preconception carrier screening (S2.5-2.7). We forecast changes in health system costs and disability-adjusted life years (DALYs) prevented as a result of population genomic screening, compared with targeted testing. Cost-effectiveness was calculated from a health system payer perspective using incremental cost-effectiveness ratio (ICER $=\Delta$ costs/ $\triangle D A L Y s)$ for each independent model, then combined for all models. For detailed methods and tree model diagrams see Supplementary Materials S2.1-2.7. CF cystic fibrosis, FXS fragile X syndrome, SMA spinal muscular atrophy.

surveillance, and interventions for all identified pathogenic variant carriers. Incremental cost-effectiveness ratio (ICER) was compared between population screening and targeted testing, below the willingness-to-pay (WTP) threshold of AUD $\$ 50,000 /$ DALY prevented. ${ }^{30}$ DALYs and costs for surveillance, intervention, and treatments were discounted by $3 \%$ to account for investment depreciation over time. Discounting was not applied to genomic screening, considered a fixed, up-front cost at present value. Analysis was conducted from a health-care system/payer perspective, and time horizon was lifetime. Consolidated Health Economic Evaluation Reporting Standards were followed (Supplementary Materials).

For each scenario, we calculated the following outputs compared with targeted testing (in AUD\$M): costs of up-front genomic screening and confirmation testing; subsequent downstream medical costs following screening; total incremental health system investment cost; DALYs prevented; disease cases prevented (total, per million); deaths prevented (total, per million); and ICER (\$AUD/DALY prevented). Calculations are shown as median values from 2000 model simulations for each variable. Data is not always normally distributed; therefore, combined results do not always reflect arithmetic addition of median values.

\section{Uncertainty and sensitivity analysis}

Model inputs were obtained from Australian Government sources and literature (Table S1). When uncertainty ranges were unavailable, we assumed $\pm 25 \%$ uncertainty around input parameters. Model results are presented with median and 95\% confidence intervals, with models run iteratively using 2000 simulations for each scenario. We conducted 


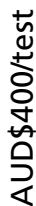

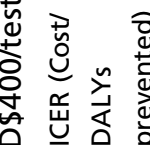

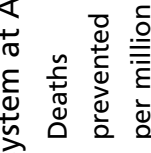

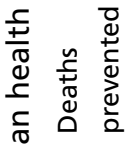

离

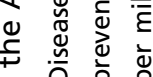

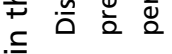

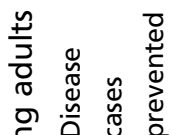

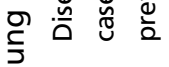

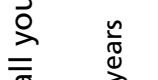

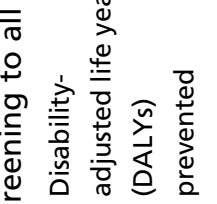

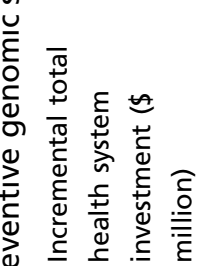

흐

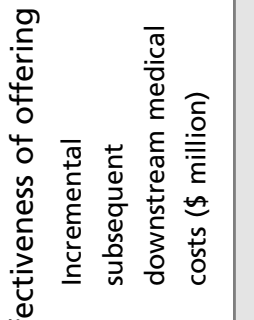

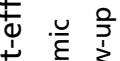

О ठำ 은

它

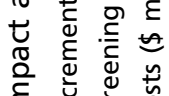

$\frac{5}{0}$
$\frac{1}{3}$
$\frac{0}{0}$
$\frac{0}{0}$
$\frac{0}{0}$

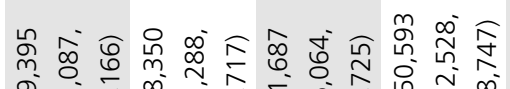

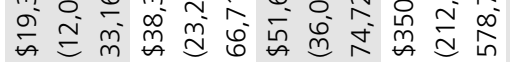

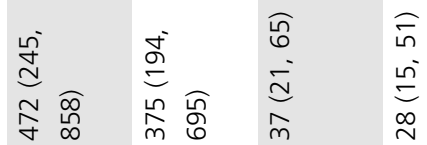

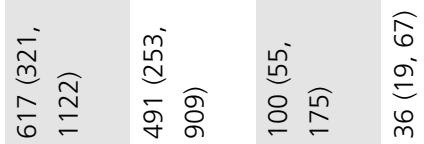

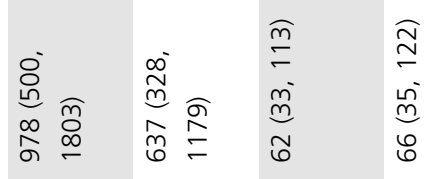

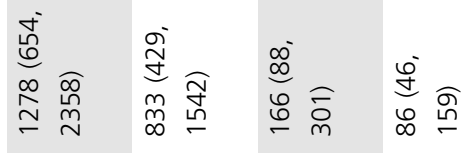

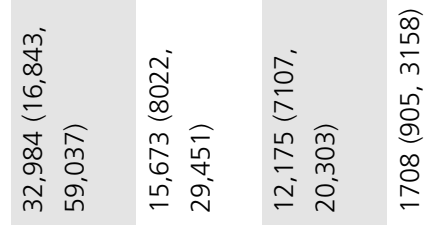

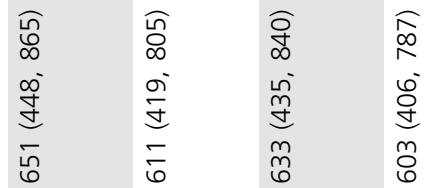

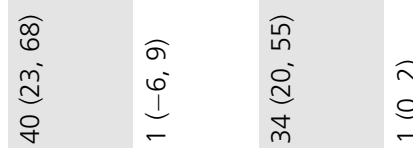

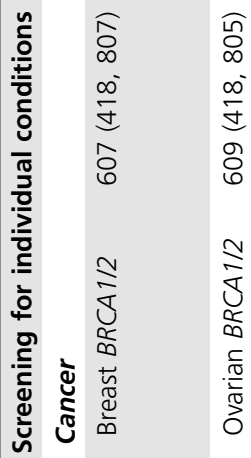

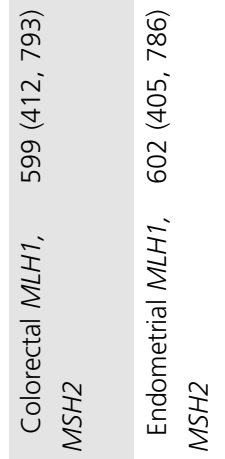

商

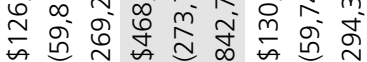

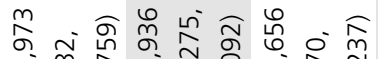

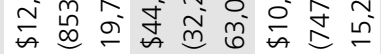

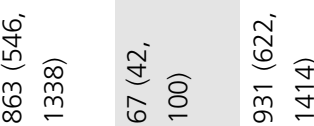

I $\quad \dot{0} \quad$ के

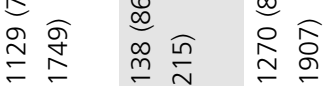

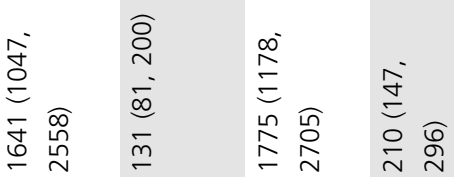

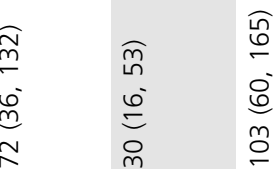

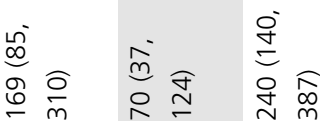

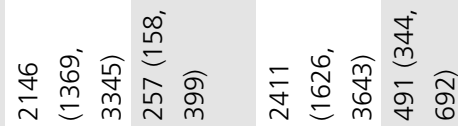

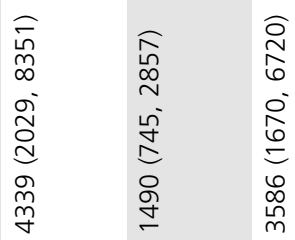

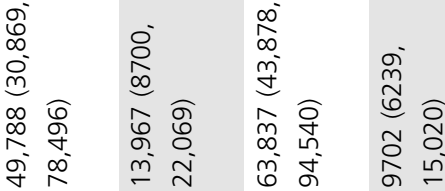

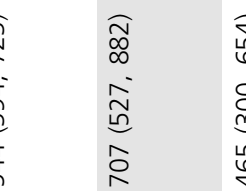

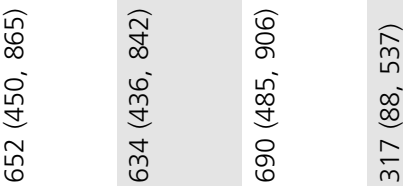
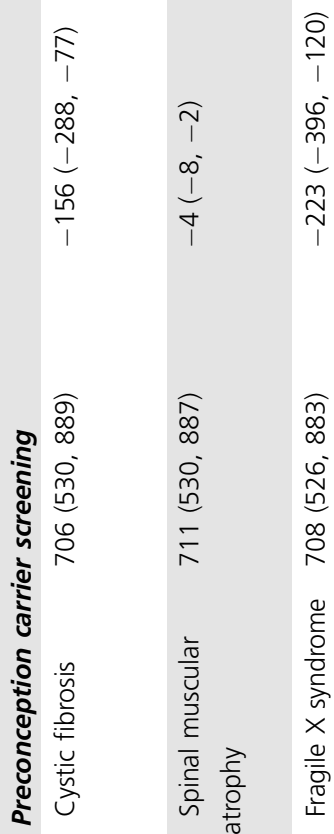

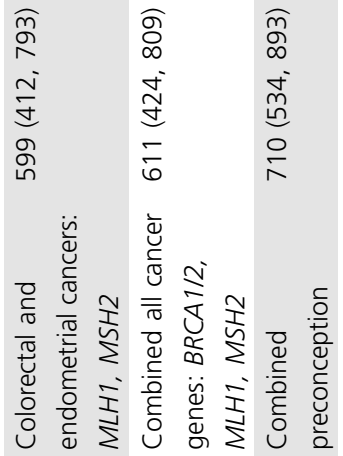




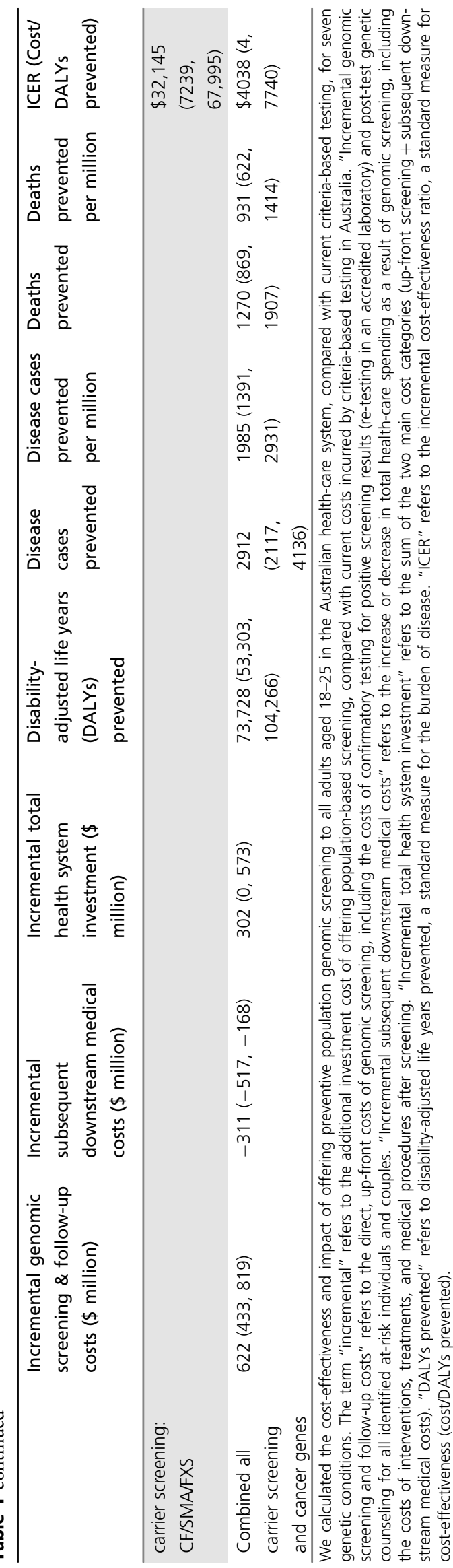

probabilistic sensitivity analyses to explore robustness on cost-effectiveness planes, with a WTP threshold of $\$ 50,000 /$ DALY prevented. We conducted one-way sensitivity analysis to investigate variations in ICER related to key variables/ assumptions.

\section{RESULTS}

Burden of disease under current rates of testing

Under current rates of targeted testing and treatment regimens, we forecast 8473 (6484 to 11,118) cancers occurring in the target population due to autosomal dominant pathogenic variants (PVs) in BRCA1/BRCA2/MLH1/MSH2. This was estimated to result in 4082 (3143 to 5313) cancer deaths.

The number of all-cause cancers (suffered by both PV carriers and non-PV carriers) forecast in the population was $416,308$ ( 347,575 to 489,044$)$, leading to 178,439 (148,952 to $209,164)$ cancer deaths. Therefore, under the current model of targeted testing, we estimated cancers attributable to BRCA1/ BRCA2/MLH1/MSH2 account for $2.0 \%(1.5-2.8 \%)$ of all cancers and $2.3 \%(1.7-3.1 \%)$ of cancer deaths in the population. We forecast 1988 (1760 to 2216) births affected by CF/SMA/FXS.

The total disease burden in the modeled population, under current targeted testing, was estimated at 1.85 million DALYs (1.53 to 2.18 million) due to all-cause cancer, of which $143,123(111,026$ to 184,988$)$ were due to BRCA1/BRCA2/ MLH1/MSH2, and 59,332 (50,672 to 68,755) due to CF/SMA/ FXS (Table S5).

\section{Impact of population genomic screening}

With an assumed $71 \%$ testing uptake and conservative intervention estimates, we forecast population-based cancer gene testing would reduce the number of cancers due to BRCA1/BRCA2/MLH1/MSH2 PVs by $28.8 \%$ (20.6-39.6\%), preventing 2411 (1626 to 3643) cancers. Cancer deaths would reduce by $31.2 \%$ (22.6\% to $42.4 \%$ ), preventing 1270 (869 to 1907) deaths, compared with targeted testing. We estimated cancers attributable to BRCA1/BRCA2/MLH1/MSH2, as a proportion of all cancers, would reduce to $1.5 \%(1.0-2.1 \%)$, with attributable cancer deaths reducing to $1.6 \%(1.1-2.2 \%)$ of total. Screening was estimated to prevent $63,837(43,878$ to $94,540)$ cancer-related DALYs, reducing the total cancer burden in the population by $3.5 \%(2.3-5.2 \%)$. Preconception carrier screening was estimated to reduce the number of disease cases of CF/SMA/FXS by $24.8 \%(17.6-34.1 \%)$ to avert 491 (344 to 692) cases (Table 1).

\section{Cost-effectiveness of population genomic screening}

We calculated the cost-effectiveness of population genomic screening using a per-test cost of AUD $\$ 400$ and WTP threshold of $\$ 50,000 / \mathrm{DALY}$, compared with targeted testing. We first investigated whether population screening for any one of the seven conditions alone was cost-effective in isolation, above targeted testing (Table S5). Preconception carrier screening for any single condition was not found to be cost-effective (Table 1). 
a

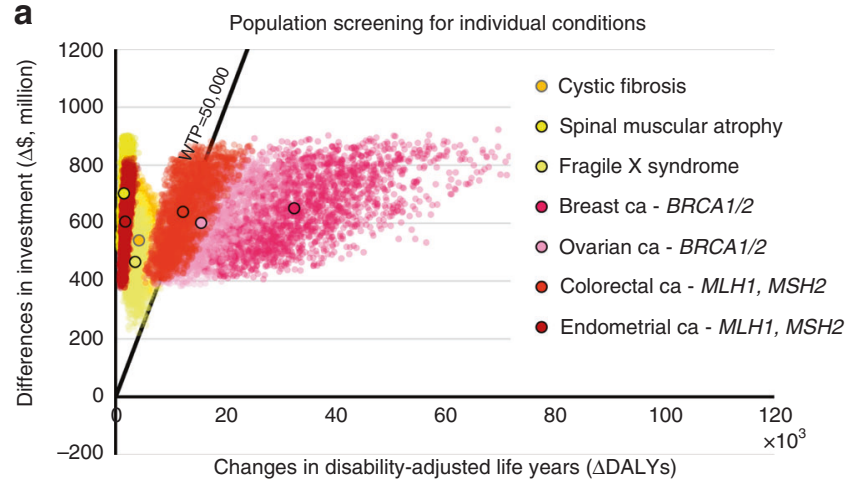

C

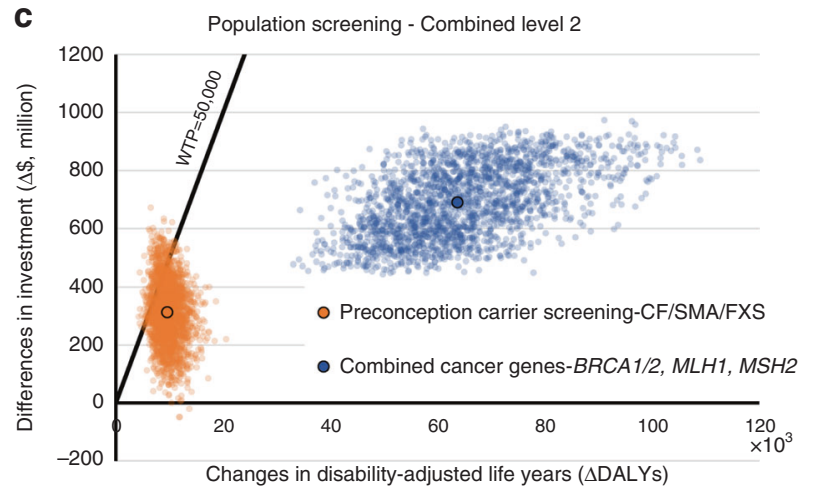

b

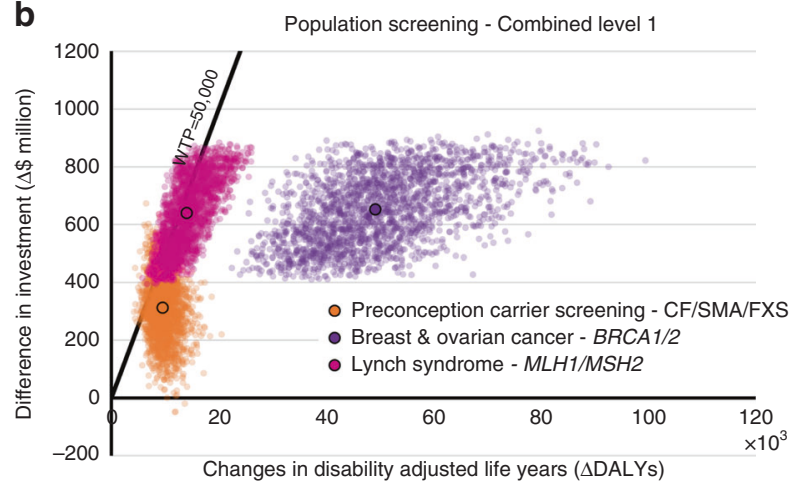

d

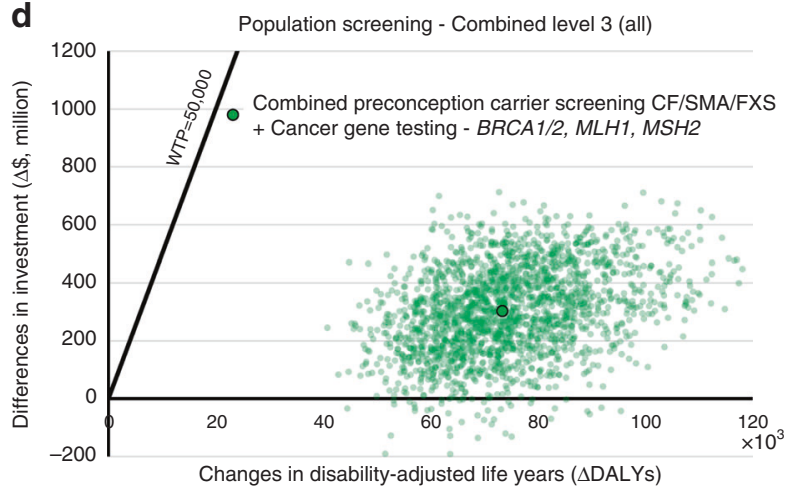

Fig. 3 Preventive genomic screening of young adults becomes increasingly cost-effective as more conditions are screened for concurrently. Probability sensitivity analysis was used to calculate the cost-effectiveness of preventive genomic screening in early adulthood at a fixed per-test cost of AUD $\$ 400$, versus targeted testing. Population cancer gene testing was modeled for four familial cancers-breast, ovarian, colorectal, and endometrialattributable to $B R C A 1, B R C A 2, M L H 1$, and MSH2 variants, plus preconception carrier screening (PCS) for three severe rare genetic conditions (cystic fibrosis $[\mathrm{CF}]$, spinal muscular atrophy [SMA], and fragile $X$ syndrome [FXS]). Cost-effectiveness was calculated for each condition independently and then in combinations. $Y$-axis shows differences in investment (cost) in \$AUDM, and $X$-axis shows changes in disability-adjusted life years (DALYs). Cost-effectiveness is represented as the incremental cost-effectiveness ratio (ICER), or cost/DALY prevented. ICER under the willingness-to-pay threshold of AUD $\$ 50,000 / D A L Y$ is considered cost-effective. Models were run iteratively using 2000 simulations for each scenario, with $\pm 25 \%$ uncertainty ranges, each iteration represented as a separate dot. (a) Independent population screening for each of the seven conditions. (b) Combined level 1: population cancer gene testing (breast and ovarian, Lynch syndrome); and population PCS (CF + SMA + FXS) separately. (c) Combined level 2: population testing for all cancer genes together (BRCA1/ 2, MLH1, MSH2); and population PCS (CF + SMA + FXS). (d) Combined level 3: concurrent population genomic screening for all seven conditions combined.

However, consistent with others ${ }^{13}$ we found populationbased $B R C A 1 / 2$ testing for breast cancer alone was costeffective, above prevention currently achieved by targeted testing $\quad($ ICER $=$ AUD $\$ 12,973 \quad[\$ 8532$ to $\$ 19,759] /$ DALY prevented). Population-based BRCA1/2 testing for ovarian cancer alone was found to be less cost-effective (ICER $=$ AUD $\$ 38,350$ [ $\$ 23,288$ to $\$ 66,717] /$ DALY prevented). Screening for colorectal or endometrial cancer alone $(M L H 1 / M S H 2)$ was not cost-effective (Fig. 3a).

Secondly, we combined models to investigate the costeffectiveness of screening for multiple groups of conditions concurrently, including combined testing for hereditary breast and ovarian cancer, combined testing for Lynch syndrome, and combined carrier screening (CF + SMA + FXS) (Fig. 3b). We found all three of these combined scenarios to be costeffective, demonstrating the efficiency of combined screening. We found population testing for all four cancers combined would be highly cost-effective, independently of carrier screening (ICER AUD\$10,656 [ $\$ 7470$ to $\$ 15,237$ ]/DALY prevented) (Fig. 3c).

Finally, we considered the total health impact and costeffectiveness of combining all seven models, maintaining a combined test cost of AUD $\$ 400$ (Fig. 3d). This scenario, using conservative intervention estimates, was found to be highly cost-effective (ICER $=$ AUD $\$ 4038$ [ $\$ 4$ to $\$ 7740] /$ DALY). Screening for all seven conditions at AUD $\$ 400$ would require an additional health system investment of AUD $\$ 622$ million ( $\$ 433$ to $\$ 819$ million), above the current cost of targeted testing in Australia. This would include costs of initial screening, plus subsequent confirmatory testing and genetic counseling, for all identified PV carriers and at-risk couples in the target population. Screening would prevent an estimated $73,728(53,303$ to 104,266$)$ DALYs and save AUD $\$ 311$ million ( $\$ 168$ to $\$ 517$ million) in treatment costs through prevention, for a net health system cost of AUD $\$ 302$ million ( $\$ 0$ to $\$ 573$ million), above current expenditure (Table 1). 


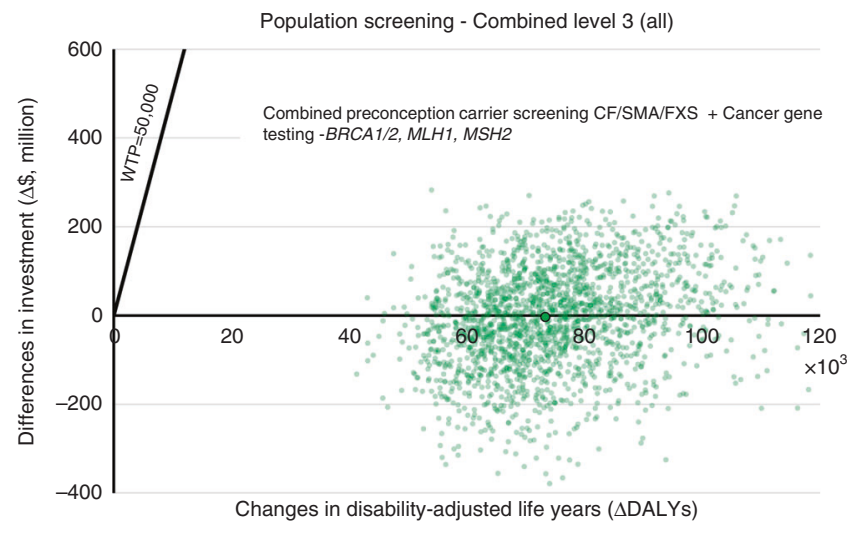

Fig. 4 Preventive genomic screening of young adults at a per-test cost of AUD $\$ 200$ would potentially be cost-saving to the health system. Probability sensitivity analysis was used to calculate the costeffectiveness of preventive genomic screening in early adulthood for seven conditions concurrently, at a per-test cost of AUD $\$ 200$, versus targeted testing. $Y$-axis shows differences in investment (cost) in \$AUDM, and $X$-axis shows changes in disability-adjusted life years (DALYs). Cost-effectiveness is represented as incremental cost-effectiveness ratio (ICER), or cost/DALY gained. ICER under the willingness-to-pay threshold of AUD $\$ 50,000 / D A L Y$ is considered cost-effective; under AUD $\$ 0 / D A L Y$ is considered cost-saving. Models were run iteratively using 2000 simulations for each scenario, with $\pm 25 \%$ uncertainty ranges, with each iteration represented as a separate dot.

\section{Probably sensitivity analysis and cost-effectiveness acceptability}

Cost-effectiveness of population screening would be highly dependent on the per-sample test cost. Therefore, we calculated cost-effectiveness at per-test costs ranging from AUD $\$ 200$ to $\$ 1200$ (Table S6, Figure S3.1-3.4). Combined cancer gene testing alone was found to be cost-effective in all scenarios, up to and including AUD\$1200 per test, demonstrating the efficiency of population-based cancer testing.

Combined screening for all seven conditions together was found to be cost-effective up to and including AUD $\$ 1200$ (Figure S3). Notably, if a combined per-test cost of AUD\$200 could be achieved, the cost of up-front screening, counseling and ongoing surveillance would reduce to AUD\$324 million ( $\$ 225$ million to $\$ 418$ million). This is roughly equivalent to the total saved medical costs through prevention in the population lifespan (AUD\$315 million [ $\$ 168$ million to $\$ 519$ million]), making screening extremely cost-effective, approaching cost-saving, for the health-care system $(\mathrm{ICER}=\mathrm{AUD} \$ 22 \quad[\$-3450$ to $\$ 2622] / \mathrm{DALY}$ prevented) (Fig. 4).

To assess impact on cost-effectiveness, we conducted oneway sensitivity analyses on the uptake of genomic screening (50-90\%); risk-reducing surgeries (90\%); prenatal diagnosis (70-90\%); reimbursement of SMA drug nusinersen (AUD $\$ 976,000$ per year, per SMA patient); and reimbursement of breast reconstruction (per risk-reducing mastectomy). In each scenario, the ICER of screening never exceeded AUD $\$ 50,000 /$ DALY. In most cases, changes made the ICER more costeffective (Table S7).

\section{DISCUSSION}

Offering population genomic screening for disease prevention is an emerging consideration for single-payer or state-funded health-care systems. For the first time, we calculated the impact and cost-effectiveness of offering health system-funded preventive genomic screening for multiple conditions to all young adults. Our results demonstrated that population screening could significantly reduce the incidence and mortality of hereditary cancers, and the burden of severe childhood-onset genetic disease, compared with targeted testing. Screening at a cost of AUD $\$ 400$ was found to be highly cost-effective. However, if AUD $\$ 200$ per-test could be achieved, screening could become cost-saving for the health system, providing a significant platform for the consideration of health system-funded screening.

Our model consistently demonstrated cost-effectiveness at per-test costs up to AUD $\$ 1200$, despite all estimates being purposely conservative. We modeled screening of only four genes for two cancer syndromes, excluding many less penetrant genes (e.g., RAD51C, RAD51D, BRIP1, PALB2, $M S H 6, P M S 2, E P C A M)$. Future model iterations will expand to include other genes and conditions. We also excluded rarer types of cancer associated with BRCA1/2 (e.g., peritoneal, male breast, prostate, pancreatic) and MLH1/MSH6 (e.g., stomach, intestine, liver, gallbladder).

We also excluded many conditions potentially amenable to population screening, such as Tay-Sachs, thalassemia, sickle cell anemia, and hereditary hemochromatosis (carrier screening), and familial hypercholesterolemia, long QT syndrome, and cardiomyopathies (adult gene testing). Many factors contributed to this decision, such as reduced penetrance and prevalence of some conditions, lack of available population health data, and existing screening programs in some cases. Over time, as the availability of information improves, further modeling may incorporate these conditions and screening scenarios.

Many of the secondary or added benefits of population screening were not modeled. These include, most notably, the impact of cascade testing of at-risk relatives after the initial screening and returning BRCA1/2 results to males. The uptake of cascade testing in Australia has been estimated at around three relatives per index case, ${ }^{31}$ which if applied at the population level, would have considerable preventive impact. The increases in economic output as a result of prevention, were also not modeled.

Conservative health cost estimates were used throughout, particularly for cancer treatment, averaged to AUD $\$ 33,400$ / year for simplicity across all invasive cancers. This likely underestimated the cost of cancer treatments, and costsavings associated with early intervention and prevention. The costs of CF/SMA/FXS treatment were also estimated conservatively, likely understating the health economic cost of these conditions, which are difficult to estimate. ${ }^{32}$

Use of preimplantation genetic diagnosis (PGD) was not modeled, despite its potential to avoid the implantation of embryos affected by genetic conditions, or predisposed to 
cancer. PGD is not currently reimbursed by the Australian system, and insufficient data exist to inform uptake rates of PGD for at-risk couples.

With regard to pregnancies affected by a genetic condition identified through population carrier screening, we modeled the decision to terminate affected pregnancies conservatively (0.50). This is despite the literature suggesting rates above 0.90 for elective TOP for conditions such as Down syndrome ${ }^{33}$ and SMA. ${ }^{34}$ We recognize this issue is controversial, and that laws and ethical positions vary considerably between countries/ jurisdictions. Variations in population attitudes based on age, religion, and other factors, as well as the criticality of preserving individual choice, were acknowledged in adopting this highly conservative estimate.

A number of additional ethical implications must be considered. Associating financial investments with the prevention of disease, especially where reproductive decisions are involved, requires sensitivity, caution, and ethical rigor. Funding decisions based on imputed cost-savings must not result in implicit pressure on individuals to violate personal ethics to reduce financial burden on society. As discussions regarding prenatal testing have demonstrated, ${ }^{35}$ there is risk that "routinization" of testing may lead to social or medical expectations of testing in all eligible individuals. These expectations, if linked with financial incentives for the health system, could risk applying implicit pressure on serious, and potentially irreversible, personal decisions. Such expectations, if applied at the population level, could risk becoming normalized, compromising the values of informed consent and individual autonomy.

To acknowledge these considerations, we used highly conservative intervention uptake estimates, especially around reproductive decisions. We strongly believe that any decision regarding individual health interventions should be grounded in informed consent and personal autonomy, without presumed obligation or implicit pressure. This should be a fundamental principle of screening, especially when personal decisions are involved in reproductive planning or prophylactic surgery.

In addition to the potential for encroaching on individual autonomy, population screening may also risk increasing the propensity for genetic discrimination, marginalization, or stigmatization. Appropriate regulatory protection, community education, and policy must be in place prior to consideration of screening. Other issues, such as increasing out-of-pocket costs and compromising access to life insurance products, ${ }^{36,37}$ are also concerns.

The model does not consider the costs and challenges of achieving informed consent at scale through public education, online resources, or other mechanisms. This would be essential. Increasing education and genomic literacy is one of the most significant challenges regarding the feasibility and appropriateness of population genomic screening. A detailed discussion of this complex and emerging challenge is beyond the scope of this CEA, yet we acknowledge its importance and consideration elsewhere. ${ }^{38}$
Our CEA has a number of limitations. First, the model was complex, with over 150 variables, meaning sections were necessarily simplified. For example, all stage 1-4 cancers were classified as "invasive," with equivalent treatment costs across all cancer types. The vast majority of model parameters were based on published literature (Table S1-S3). However, a small number (5 variables), were based on expert opinion, due to lack of available data. For these variables, estimates were kept conservative, informed by clinical practice and modeled using 2000 repeating simulations with wide uncertainty ranges $( \pm 25 \%)$.

We assumed $71 \%$ screening uptake based on one of the few randomized controlled trials involving population-based testing. ${ }^{39}$ The same figure, taken from a study in the Ashkenazi Jewish population, was recently used in CEA for population-based breast cancer testing in the UK/US general female population. ${ }^{13}$ Given population screening is not yet common, it remains to be seen whether this figure is representative. To address this uncertainty, we modeled uptake using one-way sensitivity analysis down to $50 \%$ (Table S7). We found that even at 50\% uptake, the ICER for population screening remained highly cost-effective. In fact, cost-effectiveness increased as uptake decreased (ICER = AUD\$3980/DALY at 50\%, versus $\$ 4038 / \mathrm{DALY}$ at $71 \%)$. We also assumed cancer risk in PV carriers without family history was equivalent (equal penetrance) to those identified through targeted clinical testing with family history, which is uncertain. Uptake of preventive interventions may also vary in the general population compared with affected families with disease history.

We excluded breast reconstruction following risk-reducing mastectomy. Currently, the provision and funding of breast reconstruction in Australia is inconsistent. Differences exist in access, uptake, and availability between the public and private health systems. For all aspects of this study, we made the decision to exclude data where significant disparities in access or potential for inequity exist. We modeled the cost of breast reconstruction in sensitivity analysis, and the impact on costeffectiveness was minimal (Table S7).

Resourcing and scalability of clinical services is another significant challenge with the prospect of population-based screening. Amongst the many related challenges of service delivery, we highlight training of the medical workforce, expanding clinical genetic services, achieving genetic counseling and informed consent at scale, ensuring availability of ongoing surveillance/interventions, and reducing wait times for elective procedures (i.e., colonoscopy, prophylactic surgery). Addressing these and other practical challenges would require a substantial increase in the resourcing and efficiency of genetic and other health services.

Despite many challenges related to the implementation, ethics, and feasibility of population genomic screening, our model demonstrates significant cost-effectiveness and preventive potential for this approach. We demonstrated this using only a limited set of genes and conditions, using conservative intervention uptake estimates, following current 
standard of care and publicly funded health services. Therefore, if population-based testing for a single genetic condition or application is undertaken in the future, careful consideration should be given to expanded screening for multiple conditions concurrently, to maximize cost-effectiveness and preventive impact.

Single-payer or public health-care systems, such as Australia's, have the unique opportunity to consider offering genomic screening to adults for disease prevention. Screening could follow established principles and frameworks. ${ }^{40}$ Considering population genomics from the context of public health screening could help to deliver a level of quality control, standardization, and equity of access that may not be achievable in other health systems. However, the many possible benefits of prevention will only be achievable with adequate health service scalability, public education, and ethical oversight.

\section{SUPPLEMENTARYT INFORMATION}

The online version of this article (https://doi.org/10.1038/s41436019-0457-6) contains supplementary material, which is available to authorized users.

\section{AUTHOR CONTRIBUTIONS}

$\mathrm{PL}$ conceived the study, provided leadership in executing the study, and led writing of the manuscript. PL and $L Z$ designed the study, reviewed the literature, collected data, and interpreted the results. LZ led the modeling analyses, with assistance from YB and $M R$, who generated results, wrote code and developed figures. Clinical expertise were provided by DJA for preconception carrier screening, and AH, PAJ, and IW for cancer. DJA, AH, PAJ, LP, IW, $\mathrm{DL}$ and $\mathrm{MN}$ provided critical review of the manuscript. JT provided input on ethical and genetic counseling issues, and helped write and review the manuscript. XZ and JJMc provided epidemiological and methodological oversight.

\section{DISCLOSURE}

The authors declare no conflicts of interest.

Publisher's note: Springer Nature remains neutral with regard to jurisdictional claims in published maps and institutional affiliations.

\section{REFERENCES}

1. Schwartz MLB, McCormick CZ, Lazzeri AL, et al. A model for genomefirst care: returning secondary genomic findings to participants and their healthcare providers in a large research cohort. Am J Hum Genet. 2018;103:328-337.

2. Alver $M$, Palover $M$, Saar $A$, et al. Recall by genotype and cascade screening for familial hypercholesterolemia in a population-based biobank from Estonia. Genet Med. 2018 Oct 1; 10.1038/s41436-0180311-2 [Epub ahead of print].

3. Di Marco M, D'Andrea E, Panic N, et al. Which Lynch syndrome screening programs could be implemented in the "real world"? A systematic review of economic evaluations. Genet Med. 2018;20:1131-1144.

4. Manchanda R, Legood R, Burnell $M$, et al. Cost-effectiveness of population screening for BRCA mutations in Ashkenazi Jewish women compared with family history-based testing. I Natl Cancer Inst. 2015;107:380
5. Manchanda R, Patel S, Antoniou AC, et al. Cost-effectiveness of population based BRCA testing with varying Ashkenazi Jewish ancestry. Am J Obstet Gynecol. 2017;217:578e1-e12.

6. Norman R, van Gool K, Hall J, et al. Cost-effectiveness of carrier screening for cystic fibrosis in Australia. J Cyst Fibros. 2012;11:281-287.

7. Gabai-Kapara E, Lahad A, Kaufman B, et al. Population-based screening for breast and ovarian cancer risk due to BRCA1 and BRCA2. Proc Natl Acad Sci U S A. 2014:111:14205-14210.

8. Metcalfe KA, Poll A, Royer R, et al. Screening for founder mutations in BRCA1 and BRCA2 in unselected Jewish women. J Clin Oncol. 2010;28:387-391

9. Manchanda R, Blyuss O, Gaba F, et al. Current detection rates and timeto-detection of all identifiable BRCA carriers in the Greater London population. J Med Genet. 2018;55:538-545. https://doi.org/10.1136/ jmedgenet-2017-105195, Epub 5 Apr. 2018.

10. Australia LS Misdiagnosed, misunderstood and missing out: Lynch syndrome Australia's untold health story. 2017. https://lynchsyndrome. org.au/wp-content/uploads/2017/03/Lynch-Syndrome-Report.pdf. Accessed 14 February 2019.

11. Burn J, Gerdes AM, Macrae F, et al. Long-term effect of aspirin on cancer risk in carriers of hereditary colorectal cancer: an analysis from the CAPP2 randomised controlled trial. Lancet. 2011;378:2081-2087.

12. Petelin $\mathrm{L}$, Trainer $\mathrm{AH}$, Mitchell $\mathrm{G}$, et al. Cost-effectiveness and comparative effectiveness of cancer risk management strategies in BRCA1/2 mutation carriers: a systematic review. Genet Med. 2018;20: $1145-1156$.

13. Manchanda R, Patel $S$, Gordeev VS, et al. Cost-effectiveness of Population-Based BRCA1, BRCA2, RAD51C, RAD51D, BRIP1, PALB2 Mutation Testing in Unselected General Population Women. J Natl Cancer Inst. 2018;110:714-725. https://doi.org/10.1093/jnci/djx265. Accessed 14 February 2019.

14. Manickam K, Buchanan, A, Schwartz, M, et al. Exome Sequencing-Based Screening for BRCA1/2 Expected Pathogenic Variants Among Adult Biobank Participants. JAMA Netw Open. 2018;1:e182140. https://doi.org/10.1001/jamanetworkopen.2018.2140. Accessed 14 February 2019.

15. Rowley SM, Mascarenhas L, Devereux L, et al. Population-based genetic testing of asymptomatic women for breast and ovarian cancer susceptibility. Genet Med. 2018 Sep 26; 10.1038/s41436-018-0277-0 [Epub ahead of print].

16. Dinh TA, Rosner BI, Atwood JC, et al. Health benefits and costeffectiveness of primary genetic screening for Lynch syndrome in the general population. Cancer Prev Res. 2011;4:9-22.

17. Bennette CS, Gallego CJ, Burke W, et al. The cost-effectiveness of returning incidental findings from next-generation genomic sequencing Genet Med. 2015;17:587-595.

18. Azimi $M$, Schmaus $K$, Greger $V$, et al. Carrier screening by nextgeneration sequencing: health benefits and cost effectiveness. Mol Genet Genomic Med. 2016;4:292-302.

19. Molster CM, Lister K, Metternick-Jones S, et al. Outcomes of an international workshop on preconception expanded carrier screening: some considerations for governments. Front Public Health. 2017;5:25.

20. Grody WW, Thompson BH, Gregg AR, et al. ACMG position statement on prenatal/preconception expanded carrier screening. Genet Med. 2013;15:482-483.

21. Delatycki MB, Burke J, Christie L, et al. Human Genetics Society of Australasia position statement: population-based carrier screening for cystic fibrosis. Twin Res Hum Genet. 2014;17:578-583.

22. The Human Genetics Commission, UK. Commission HG Increasing options, informing choice: a report on preconception genetic testing and screening. Crown Copyright 2010; London, 2011. https://tegalsi. hypotheses.org/files/2011/04/2011.HGC_.-Increasing-options-informingchoice-final1.pdf. Accessed 14 February 2019.

23. Archibald $A D$, Smith MJ, Burgess $T$, et al. Reproductive genetic carrier screening for cystic fibrosis, fragile $\mathrm{X}$ syndrome, and spinal muscular atrophy in Australia: outcomes of 12,000 tests. Genet Med. 2018;20: 513-523.

24. Maxwell KN, Domchek SM, Nathanson KL, Robson ME. Population frequency of germline BRCA $1 / 2$ mutations. J Clin Oncol. 2016;34:4183-4185.

25. Win AK, Jenkins MA, Dowty JG, et al. Prevalence and penetrance of major genes and polygenes for colorectal cancer. Cancer Epidemiol Biomarkers Prev. 2017;26:404-412. 
26. Kuchenbaecker KB, McGuffog $L$, Barrowdale D, et al. Evaluation of polygenic risk scores for breast and ovarian cancer risk prediction in BRCA1 and BRCA2 mutation carriers. J Natl Cancer Inst. 2017;109. https://doi.org/10.1093/jnci/djw302. Accessed 14 February 2019.

27. Dowty JG, Win AK, Buchanan DD, et al. Cancer risks for MLH1 and MSH2 mutation carriers. Hum Mutat. 2013;34:490-497.

28. Global Burden of Disease Collaborative Network. Global Burden of Disease Study 2016 (GBD 2016). Cause-specific mortality 1980-2016. Seattle, WA: Institute for Health Metrics and Evaluation (IHME); 2017.

29. Little SE, Janakiraman $V$, Kaimal $A$, et al. The cost-effectiveness of prenatal screening for spinal muscular atrophy. Am J Obstet Gynecol. 2010:202:253 e1-7.

30. Neumann PJ, Cohen JT, Weinstein MC. Updating cost-effectiveness-the curious resilience of the $\$ 50,000-$ per-QALY threshold. N Engl J Med. 2014;371:796-797.

31. Tuffaha HW, Mitchell A, Ward RL, et al. Cost-effectiveness analysis of germ-line BRCA testing in women with breast cancer and cascade testing in family members of mutation carriers. Genet Med. 2018;20:985-994.

32. Lacaze $\mathrm{P}$, Millis N, Fookes $\mathrm{M}$, et al. Rare disease registries: a call to action. Intern Med J. 2017;47:1075-1079.

33. Jacobs M, Cooper SA, McGowan R, et al. Pregnancy outcome following prenatal diagnosis of chromosomal anomaly: a record linkage study of 26,261 pregnancies. PLoS One. 2016;11:e0166909.

34. Su YN, Hung CC, Lin SY, et al. Carrier screening for spinal muscular atrophy (SMA) in 107,611 pregnant women during the period 20052009: a prospective population-based cohort study. PLoS One. 2011;6: e17067.

35. Deans Z, Newson AJ. Should non-invasiveness change informed consent procedures for prenatal diagnosis? Health Care Anal. 2011;19:122-132.

36. Newson AJ, Tiller J, Keogh LA, et al. Genetics and insurance in Australia: concerns around a self-regulated industry. Public Health Genomics. 2017:20:247-256
37. Tiller J, Otlowski M, Lacaze P. Should Australia ban the use of genetic test results in life insurance? Front Public Health. 2017;5:330.

38. Prince $A E$, Berg JS, Evans JP, et al. Genomic screening of the general adult population: key concepts for assessing net benefit with systematic evidence reviews. Genet Med. 2015;17:441-443.

39. Manchanda R, Loggenberg K, Sanderson S, et al. Population testing for cancer predisposing BRCA1/BRCA2 mutations in the Ashkenazi-Jewish community: a randomized controlled trial. J Natl Cancer Inst. 2015;107:379.

40. Andermann A, Blancquaert I, Beauchamp S, Dery V. Revisiting Wilson and Jungner in the genomic age: a review of screening criteria over the past 40 years. Bull World Health Organ. 2008;86:317-319.

(i) Open Access This article is licensed under a Creative Commons Attribution-NonCommercial-NoDerivatives 4.0 International License, which permits any non-commercial use, sharing, distribution and reproduction in any medium or format, as long as you give appropriate credit to the original author(s) and the source, and provide a link to the Creative Commons license. You do not have permission under this license to share adapted material derived from this article or parts of it. The images or other third party material in this article are included in the article's Creative Commons license, unless indicated otherwise in a credit line to the material. If material is not included in the article's Creative Commons license and your intended use is not permitted by statutory regulation or exceeds the permitted use, you will need to obtain permission directly from the copyright holder. To view a copy of this license, visit http://creativecommons.org/licenses/by-nc-nd/4.0/.

(c) The Author(s) 2019 


\section{University Library}

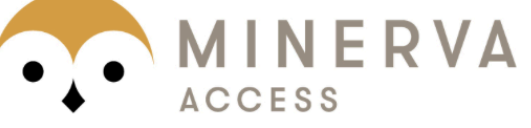

A gateway to Melbourne's research publications

Minerva Access is the Institutional Repository of The University of Melbourne

\section{Author/s:}

Zhang, L;Bao, Y;Riaz, M;Tiller, J;Liew, D;Zhuang, X;Amor, DJ;Huq, A;Petelin, L;Nelson, M;James, PA;Winship, I;McNeil, JJ;Lacaze, P

Title:

Population genomic screening of all young adults in a health-care system: a costeffectiveness analysis

Date:

2019-09-01

Citation:

Zhang, L., Bao, Y., Riaz, M., Tiller, J., Liew, D., Zhuang, X., Amor, D. J., Huq, A., Petelin, L., Nelson, M., James, P. A., Winship, I., McNeil, J. J. \& Lacaze, P. (2019). Population genomic screening of all young adults in a health-care system: a cost-effectiveness analysis.

GENETICS IN MEDICINE, 21 (9), pp.1958-1968. https://doi.org/10.1038/s41436-019-0457-6.

Persistent Link:

http://hdl.handle.net/11343/250393

License:

CC BY-NC-ND 